Draft Version SEPTEMBer 17, 2021

Preprint typeset using $\mathrm{L}_{\mathrm{A}} \mathrm{T}_{\mathrm{E}} \mathrm{X}$ style emulateapj v. 5/2/11

\title{
EXTRAGALACTIC STAR-FORMING GALAXIES WITH HYPERNOVAE AND SUPERNOVAE AS HIGH-ENERGY NEUTRINO AND GAMMA-RAY SOURCES: THE CASE OF THE 10 TEV NEUTRINO DATA
}

\author{
Nicholas Senno $^{1}$, Peter Mészáros ${ }^{1}$, Kohta Murase ${ }^{1,2}$, Philipp Baerwald ${ }^{1}$, and Martin J. Rees ${ }^{3}$ \\ Draft version September 17, 2021
}

\begin{abstract}
In light of the latest IceCube data, we discuss the implications of the cosmic ray (CR) energy input from hypernovae and supernovae into the Universe, and their propagation in the hosting galaxies and galaxy clusters or groups. The magnetic confinement of CRs in these environments may lead to efficient neutrino production via $p p$ collisions, resulting in a diffuse neutrino spectrum extending from $\mathrm{PeV}$ down to $10 \mathrm{TeV}$ energies, with a spectrum and flux level compatible with that recently reported by IceCube. If the diffuse $10 \mathrm{TeV}$ neutrino background largely comes from such CR reservoirs, the corresponding diffuse $\gamma$-ray background should be compatible with the recent Fermi data. In this scenario, the CR energy input from hypernovae should be dominant over that of supernovae, implying that the starburst scenario does not work if the supernova energy budget is a factor of two larger than the hypernova energy budget. Thus, this strong case scenario can be supported or ruled out in the near future.

Subject headings: cosmic rays — galaxies: clusters: general — neutrinos — supernovae: general
\end{abstract}

\section{INTRODUCTION}

The detection of $\mathrm{PeV}$ and sub-PeV astrophysical neutrinos by IceCube (Aartsen et al. 2013: IceCube Collaboration 2013 Aartsen et al. 2014b), which more recently has been extended down to $10 \mathrm{TeV}$ energies (Aartsen et al. 2015), is a major development. The origin of these neutrinos is a matter of intense interest (for recent reviews, see e.g., Mészáros 2014; Murase 2014). Star-forming galaxies, especially starbursts, are promising candidates (Loeb \& Waxman 2006, Murase et al. 2013; Katz et al. 2013; Tamborra et al. 2014 Chang \& Wang 2014 Anchordoqui et al. 2014), in which the major contributing sources may be supernovae ( $\mathrm{SNe}$ ), as well as their hyper-energetic equivalent the so-called hypernovae (HNe) (Sveshnikova 2003: Fox et al. 2013, He et al. 2013; Murase et al. 2013; Liu et al. 2014 Ahlers \& Murase 2014) or active galactic nuclei (AGN) (Murase et al. 2014, Tamborra et al. 2014). Other possible candidates include low-luminosity classes of $\gamma$-ray bursts (Murase

\begin{tabular}{|l|}
\hline nbs5044@psu.edu \\
\hline i Dept. of Physics, Dept. of Astronomy \& As- \\
trophysics, Center for Particle and Gravitational As- \\
trophysics, Pennsylvania State University, University \\
Park, PA 16802. USA \\
\hline \hline Institute for Advanced Study, Princeton, NJ 08540, \\
USA \\
3 Institute of Astronomy, University of Cambridge, \\
Cambridge CB3 0HA, U.K.
\end{tabular}

\& Ioka 2013; Liu \& Wang 2013), radio-loud active galactic nuclei (Stecker et al. 1991; AlvarezMuñiz \& Mészáros 2004; Murase et al. 2014; Dermer et al. 2014 Kalashev \& Troitsky 2014), galaxy clusters and groups (Murase et al. 2008a. 2013) with accretion shocks (Keshet et al. 2004. Inoue et al. 2005) that may accelerate cosmic rays (CRs) to higher energies (Hillas 1984; Biermann \& Strittmatter 1987) or other CR sources such as galaxy mergers in clusters (Kashiyama \& Mészáros 2014).

In this work, we will concentrate mainly on the $\mathrm{HN} / \mathrm{SN}$ origin of neutrinos from $p p$ interactions in the starburst and normal star-forming intragalactic material and the intracluster medium. In particular, we discuss their implications in light of the latest IceCube data in the $10 \mathrm{TeV}$ range. Constraints from the diffuse $\gamma$-ray background measured by Fermi are even more pronounced in this case.

\section{HYPERNOVA AND SUPERNOVA ENERGY INPUT RATE}

HNe typically belong to a sub-class of broadline Type $\mathrm{Ib} / \mathrm{c}$ SNe with ejecta kinetic energies of order $E_{k}=10^{52} E_{k, 52} \mathrm{erg}$, representing a fraction $\xi_{h n} \simeq 4 \times 10^{-2} \xi_{h n,-1.4}$ of all corecollapse SNe, with substantial uncertainties $(\mathrm{Ar}-$ cavi et al. 2010, Guetta \& Della Valle 2007; Smith et al. 2011). The rate of all core-collapse 
SNe is $1.06 \times 10^{-4} \mathrm{Mpc}^{-3} \mathrm{yr}^{-1}$ (e.g., Taylor et al. $2014)$, which implies a local HN rate of $\mathcal{R}_{h n} \sim$ $4 \times 10^{-6} \xi_{h n,-1.4} \mathrm{Mpc}^{-3} \mathrm{yr}^{-1}$. If the fraction of $\mathrm{HN}$ remnant kinetic energy transferred to CRs is $E_{c r, h n} \simeq 2.8 \times 10^{51}$ ergs, the CR energy density input rate in the Universe is $\dot{U}_{c r} \simeq E_{c r, h n} \mathcal{R}_{h n} \simeq$

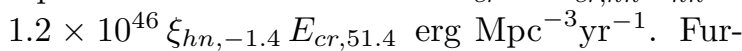
thermore, if the CRs are protons with a power law distribution $N\left(\varepsilon_{p}\right) \propto \varepsilon_{p}^{-\Gamma}$ between $\varepsilon_{p, \text { min }} \sim$ $1 \mathrm{GeV}$ and $\varepsilon_{p, \max } \lesssim 10^{17} \varepsilon_{p, 17} \mathrm{eV}$, the energy density input rate per logarithmic interval of en$\operatorname{ergy}$ is $\varepsilon_{p} Q_{\varepsilon_{p}} \simeq \dot{U}_{c r} / \mathcal{C}$ erg $\mathrm{Mpc}^{-3} \mathrm{yr}^{-1}$, where the bolometric correction is $\mathcal{C}=\ln \left(\varepsilon_{p, \max } / \varepsilon_{p, \min }\right)$ for a spectral index $\Gamma=2^{\dagger}$. Assuming $\Gamma \sim 2$ and taking $\mathcal{C} \sim 18 \mathcal{C}_{18}$ the local $(z=0)$ CR energy input per logarithmic interval in the Universe due to hypernovae is

$$
\begin{aligned}
& \left(\varepsilon_{p} Q_{\varepsilon_{p}}\right)_{h n} \simeq 6.4 \times 10^{44} \xi_{h n,-1.4} \mathcal{C}_{18}^{-1} E_{c r, h n, 51.4} \\
& \text { erg } \mathrm{Mpc}^{-3} \mathrm{yr}^{-1} \text {, }
\end{aligned}
$$

which is larger than the typical value expected for $\gamma$-ray bursts. Conventional SNe will also contribute significantly to lower-energy CRs, having a smaller kinetic energy input $E_{k, s n} \simeq$ $10^{51} E_{k s n, 51}$ erg but a larger rate $\mathcal{R}_{s n}$. The typical CR energy of SNe is uncertain and could be less (e.g. $E_{c r, s n}=4.8 \times 10^{49} \mathrm{erg}$ ). In general, the energy injection rate for $\mathrm{SNe}$ is given by

$$
\left(\varepsilon_{p} Q_{\varepsilon_{p}}\right)_{s n}=\frac{\left(1-\xi_{h n}\right)}{\xi_{h n}} \frac{\mathcal{C}_{h n}}{\mathcal{C}_{s n}} \frac{E_{c r, s n}}{E_{c r, h n}}\left(\varepsilon_{p} Q_{\varepsilon_{p}}\right)_{h n} .
$$

It is believed that SNe can typically accelerate CRs to a maximum energy $\varepsilon_{p, \text { max }} \sim 10^{15} \mathrm{eV}$ resulting in $\mathcal{C}_{s n} \sim 13.8$. The energy input due to conventional $\mathrm{SNe}$ would be typically larger than that of $\mathrm{HNe}$ at lower energies. However, with the parameters given above, Eq. (2) implies the energy input rate of SNe for CRs with $\varepsilon_{p} \lesssim \varepsilon_{p, \max , s n}$ is roughly half that of HNe. Below, we leave this ratio as a free parameter.

\section{SHOCK ACCELERATION}

A typical Type Ibc SN has a bulk ejecta mass of $M_{e j} \simeq 3 M_{e j, 0.5} M_{\odot}$, and a HN has an average velocity $\beta_{e j}=\left(V_{e j} / c\right)=6.1 \times$ $10^{-2} E_{k, h n, 52}^{1 / 2} M_{e j, 0.5}^{-1 / 2}$. The postshock random magnetic field strength is expected to be amplified to a fraction $\epsilon_{B}$ of the postshock thermal energy, $B_{s} \sim\left(16 \pi \epsilon_{B} n_{g} m_{N} c^{2} \beta_{e j}^{2}\right)^{1 / 2}$, with $n_{g}$ being the interstellar particle number density. The upstream magnetic field should also be amplified by

\footnotetext{
${ }^{\dagger}$ It would be $\mathcal{C}=\left[1-\left(\varepsilon_{p, \max } / \varepsilon_{p, \min }\right)^{2-\Gamma}\right] \varepsilon_{p, \min }^{2-\Gamma}(\Gamma-$ $2)^{-1}$ if $\Gamma \neq 2$.
}

e.g., CR-streaming instabilities, but in any case the stronger magnetic fields in starburst galaxies may also be enough (Murase et al. 2013). Diffusive shock acceleration in the blast wave leads to a power law spectrum distribution $N\left(\varepsilon_{p}\right) \propto \varepsilon_{p}^{-\Gamma}$, typically with $\Gamma \gtrsim 2$, up to a maximum energy $\varepsilon_{p, \max } \simeq(3 / 20) Z e B_{s} R_{d e c} \beta_{e j}($ Drury 1983), or

$$
\varepsilon_{p, h n, \max } \simeq 10^{17} Z n_{g, 2.3}^{1 / 6} E_{k, h n, 52} M_{e j, 0.5}^{-2 / 3} \mathrm{eV}
$$

for CRs with charge $Z$. However, in the following work we only consider CR protons. The above equation implies that the CRs are accelerated to $\sim 100 \mathrm{PeV}$ as the shock slows and enters the socalled Sedov-Taylor phase. The maximum CR energy is expected to decrease with time during this deceleration phase (Drury 2011). Also note that while many $\mathrm{SNe}$ and HNe may happen in relatively low-density regions such as superbubbles, the dependence on $n_{g}$ is weak. For normal SNe, using the same parameters except for $E_{k}=10^{51} \mathrm{erg}$, the maximum energy would be $\varepsilon_{p, s n, \text { max }} \simeq 1.1 \times 10^{16} Z n_{g, 2.3}^{1 / 6} E_{k, 51} M_{e j, 0.5}^{-2 / 3} \mathrm{eV}$. As we will show below, HNe that occur in starburst galaxies can accelerate the majority of CRs which produce detectable high-energy neutrinos. Although we use typical numbers for $\mathrm{HNe}$ for our estimates, in star-forming galaxies hosting an AGN the latter may be also contribute as a 10-100 PeV CR accelerator (Murase et al. 2014, Tamborra et al. 2014).

CRs suffer energy losses both during acceleration and after escaping their source. Synchrotron losses are negligible at the energies considered here, the dominant loss mechanism being hadronuclear $(p p)$ collisions. The effective optical depth to $p p$ collisions undergone while advected downstream of the blast wave is $\tau_{p p, s} \sim$ $t_{d y n} / t_{p p} \sim \kappa \sigma_{p p} R(c / V)$, where $\kappa \sim 0.5$ is the inelasticity and $\sigma_{p p}\left(\varepsilon_{p}=100 \mathrm{PeV}\right) \sim 10^{-25} \mathrm{~cm}^{2}$ (in the numerical calculations presented below, we use the energy dependent inelastic $p p$ cross section presented in Kelner et al. 2006). Thus $\tau_{p p, s} \sim 1.3 \times 10^{-6} E_{k, h n, 52}^{-1 / 2} M_{e j, 0.5}^{5 / 6} n_{g, 2.3}^{-1 / 3}$, which is negligible compared to losses during the subsequent propagation. Similar considerations apply also to the supernovae.

\section{PROPAGATION EFFECTS AND PP OPTICAL DEPTH}

The propagation of the CRs in the turbulent magnetic field of the host galaxy and galaxy cluster depends, in the diffusion approximation, on the strength of the magnetic field $B$, the CR Larmor radius $r_{L}$, and the coherence length $\ell_{c}$ of the magnetic field fluctuations. At the highest energies $\varepsilon_{p}$, where $r_{L}\left(\varepsilon_{p}\right) \gg \ell_{c}$, the CR diffu- 
sion coefficient is $D\left(\varepsilon_{p}\right) \propto r_{L}\left(\varepsilon_{p}\right)^{2}$. At lower energies, where $r_{L}\left(\varepsilon_{p}\right) \lesssim \ell_{c}$, the diffusion coefficient is $D\left(\varepsilon_{p}\right) \propto r_{L}\left(\varepsilon_{p}\right)^{\alpha}$, where $\alpha=1 / 3(1 / 2)$ for a Komolgoroff (Kraichnan) fluctuation power spectrum (e.g., Berezinsky et al. 1997). The two regimes can be interpolated as

$$
D\left(\varepsilon_{p}\right)=D_{*}\left[\left(\varepsilon_{p} / \varepsilon_{p, *}\right)^{\alpha}+\left(\varepsilon_{p} / \varepsilon_{p, *}\right)^{2}\right]
$$

where $r_{L}\left(\varepsilon_{p *}\right)=\ell_{c} / 5$ with $D_{*} \simeq(1 / 4) \operatorname{cr}_{L}\left(\varepsilon_{p *}\right)$ (Parizot 2004). Below we shall use $\alpha=1 / 3$ as an example, but the discussion can be generalized to a general positive $\alpha$ value.

After leaving the source (e.g. $\mathrm{HNe}$ or $\mathrm{SNe}$ ), the CRs first propagate diffusively through the host galaxy or are advected away by a strong galactic wind, with typical velocities of $V_{w} \sim$ $1500 \mathrm{~km} / \mathrm{s}$ in starburst galaxies (Strickland \& Heckman 2009) and $V_{w} \sim 500 \mathrm{~km} / \mathrm{s}$ for normal star-forming galaxies (Crocker 2012; Keeney et al. 2006). For a starburst galaxy (SBG) the gas scale height $H_{g} \sim 30-300$ pc may be parameterized as $H_{s b g} \sim 300 p c \simeq 10^{21} H_{21} \mathrm{~cm}$. We assume a magnetic field strength of $B_{g} \sim 200 \times 10^{-6} B_{g,-3.7} \mathrm{G}$ and a coherence length parameterized here as $\ell_{c, g} \sim 10^{-1} H_{g} \sim 30 \mathrm{pc} \simeq 10^{20} \ell_{g, 20} \mathrm{~cm}$. Both quantities are subject to large uncertainties and variations, so that the diffusion coefficient adopted here corresponds to the optimistic case. For our fiducial starburst galaxy parameters, we obtain $\varepsilon_{p *, g} \sim 1.11 \times 10^{18} \ell_{g, 20} B_{g,-3.7} \mathrm{eV}$ and $D_{*, g} \sim 1.4 \times 10^{29} \ell_{g, 20} \mathrm{~cm}^{2} / \mathrm{s}$. To ensure CR confinement, we require the coherence length to satisfy $\varepsilon_{p *, g} \gtrsim 10-100 \mathrm{PeV}$ (Murase et al. 2013). For a normal star-forming galaxy (SFG), we take the typical scale height to be $H_{g} \sim 1000 \mathrm{pc}$, with $\ell_{c .,} \sim 10^{-1} H_{g}$, a magnetic field of $B_{g} \sim 6 \mu \mathrm{G}$ (Beck 2008) and interstellar medium density of $n_{g} \sim 1 \mathrm{~cm}^{-3}$.

Given the above, the time for CR diffusive escape from the galaxy can be calculated, which for starburst galaxies is $t_{d, g}=H_{g}^{2} / 6 D_{g} \simeq$ $1.5 \times 10^{12} H_{g, 21}^{2} \ell_{g, 20} B_{g,-3.7}^{2} \varepsilon_{p, 17.2}^{-1 / 3} \mathrm{~s}$. The time for advective escape is $t_{w, g}=H_{g} / V_{w} \simeq 6.2 \times$ $10^{12} H_{g, 21} V_{w, 3.2}^{-1}$ s regardless of the CR energy. Notice that advective escape dominates diffusive escape from the galaxy for CRs with energy $\varepsilon_{p} \lesssim \varepsilon_{w}$ with

$$
\varepsilon_{w}=\frac{Z e B l_{c}^{1-1 / \alpha}}{5}\left(\frac{10 V_{w} H_{g}}{3 c}\right)^{1 / \alpha},
$$

yielding $\varepsilon_{w} \sim 5.1 \times 10^{15} \mathrm{eV}$ for the fiducial parameters used here.

The effective $p p$ optical depth undergone during propagation in a starburst galaxy is $\tau_{p p, g} \simeq$

$$
\begin{aligned}
n_{g} \kappa \sigma_{p p} c & \min \left[t_{d, g}, t_{w, g}\right] \text { or } \\
\tau_{p p, g} & \sim 4.9 \times 10^{-3} n_{g, 2.3} H_{g, 21}^{2} \ell_{g, 20} B_{g,-3.7}^{2} \varepsilon_{p, 19}^{-2} \\
\tau_{p p, g} & \sim 0.55 \quad n_{g, 2.3} H_{g, 21}^{2} \ell_{g, 20} B_{g,-3.7}^{2} \varepsilon_{p, 17.2}^{-1 / 3} \\
\tau_{p p, g} & \sim 1 \quad n_{g, 2.3} H_{g, 21} V_{w, 3.2}
\end{aligned}
$$

in the ranges $\left(\varepsilon_{p}>\varepsilon_{p *, g}\right),\left(\varepsilon_{w}<\varepsilon_{p}<\varepsilon_{p *, g}\right)$ and $\left(\varepsilon_{p}<\varepsilon_{w}\right)$, respectively.

One can see that starburst galaxies are efficient neutrino factories via $p p$ interactions due to their high interstellar gas density. As seen in Fig. 1-4, normal star-forming galaxies have lower values of $\varepsilon_{p *, g}, \varepsilon_{w}$, and $\tau_{p p, g}$ resulting in only a modest amount of neutrinos produced at high energies. We will show below that if the starburst fraction is high and CRs with energies up to $\sim 10-100 \mathrm{PeV}$ are sufficiently confined, the majority of the observed high-energy diffuse neutrino flux can be explained using HNe or other sources in starburst galaxies.

For the subsequent propagation in the galaxy cluster or group, the average magnetic field and coherence length are parameterized as $B_{c l} \sim 10^{-6} B_{c l,-6} \mathrm{G}$ and $\ell_{c, c l} \sim 30 \mathrm{kpc}=10^{23} \ell_{23} \mathrm{~cm}$. This implies $\varepsilon_{p *, c l} \sim 5.6 \times 10^{18} Z \ell_{23} B_{c l,-6} \mathrm{eV}$ and $D_{*, c l} \sim 1.4 \times 10^{32} Z \ell_{23} \mathrm{~cm}^{2} \mathrm{~s}^{-1}$. For a cluster of $10^{15} M_{\odot}$ the virial radius is $R_{c l} \sim 2.6 M_{15}^{1 / 3} \mathrm{Mpc} \simeq 8 \times 10^{24} M_{15}^{1 / 3} \mathrm{~cm}$ and the diffusion time is $t_{d, c l}=R_{c l}^{2} / 6 D$. At the maximum proton energy this is $t_{d, c l}\left(\varepsilon_{p, \max }\right) \sim 2.3 \times$ $10^{17} M_{15}^{1 / 3} \ell_{23}^{-2 / 3} B_{c l,-6}^{1 / 3} Z^{1 / 3} n_{c l,-4}^{-1 / 18} \epsilon_{B,-2}^{-1 / 6} E_{k, 52.5}^{-1 / 3} M_{e j, 1}^{2 / 9}$ $\varepsilon_{p, 16.94}^{-1 / 3}$ s. Similarly to the galactic component mentioned above, there is a spectral break when the diffusion time exceeds the injection time of CRs (Murase et al. 2008a). Assuming CR injection effectively occurs during the Hubble time at the corresponding redshift (i.e. $\left.t_{\text {age }}(z)=\int_{z}^{\infty} d z^{\prime}\left|\frac{d t_{*}}{d z^{\prime}}\right|\right)$, for a cluster located at redshift $z=1$ such a break occurs at an energy $\varepsilon_{p, c l} \sim 4 \times 10^{17} Z \mathrm{eV}$. If the cluster break energy is higher than the maximum $\mathrm{HNe}$ energy, CR diffusion does not significantly affect the fraction of CRs that interact in the intracluster medium. The cluster $p p$ optical depth is again $\tau_{p p, c l}=n_{c l} \kappa \sigma_{p p} c \min \left[t_{d, c l}, t_{a g e}\right]$, assuming a typical intracluster gas density $n_{c l} \simeq 10^{-4} n_{c l,-4} \mathrm{~cm}^{-3}$, at high redshifts (e.g. $z=1) t_{\text {age }} \lesssim t_{d, c l}\left(\varepsilon_{p, \max }\right)$ so that

$$
\tau_{p p, c l}\left(\varepsilon_{p, \max }\right) \sim 2.7 \times 10^{-2} n_{c l,-4}\left(t_{\text {age }} / 5.8 \mathrm{Gyr}\right)
$$

For more nearby clusters $\tau_{p p, c l}$ increases as the cluster age approaches the local Hubble time, although the density is also redshift-dependent. 
For a diffusion exponent $\alpha$ different from the value $1 / 3$ used as an example above, the values of $\tau_{p p, g}, \tau_{p p, c l, H}$, etc., are calculated similarly and are somewhat different, as can be seen in the numerical results discussed in the next section.

Further $p p$ collisions occur in the intergalactic medium after the CRs escape the cluster, but with the intergalactic target density $n_{\text {igm }}=$ $2.5 \times 10^{-7}\left(\Omega_{b} h^{2} / 0.022\right) \mathrm{cm}^{-3}$, and a total flight time limited by $t_{H} \sim 10^{10} \mathrm{yr}$, the corresponding $\tau_{p p, i g m}$ is negligible compared to the previous two contributions.

\section{DIFFUSE NEUTRINO FLUX}

When high-energy CRs undergo $p p$ interactions with the ambient intragalactic and intracluster medium, charged and neutral pions are created which subsequently decay to neutrinos and $\gamma$-rays respectively. On average, the resulting neutrino and parent $\mathrm{CR}$ energies can be related by $\varepsilon_{\nu} \sim$ $0.03-0.05 \varepsilon_{p}$. As a result, the diffuse neutrino flux (per flavor per logarithmic interval of energy) can be estimated using the CR energy injection rate similarly to what is done for GRBs (Waxman \& Bahcall 1997; Murase et al. 2013), as

$$
\varepsilon_{\nu}^{2} \Phi_{\varepsilon_{\nu}}=\frac{c}{4 \pi} \int_{0}^{z} \sum_{i} \frac{f_{i, p p}}{6} \frac{\left(\varepsilon_{p} Q_{\varepsilon_{p}}\right)_{p h y s}}{\left(1+z^{\prime}\right)^{4}}\left|\frac{d t}{d z^{\prime}}\right| d z^{\prime}
$$

where the physical CR energy injection rate per energy bandwidth at a given redshift $z$ is related to Eq. (1, 2), cosmological evolution is taken into account by the scale factor $S(z)$ so the normalized physical star formation rate is

$$
\begin{aligned}
& \left(\varepsilon_{p} Q_{\varepsilon_{p}}\right)_{\text {phys }}(z)= \\
& \quad\left[\left(\varepsilon_{p} Q_{\varepsilon_{p}}\right)_{h n}+\left(\varepsilon_{p} Q_{\varepsilon_{p}}\right)_{s n}\right](1+z)^{3} S(z)
\end{aligned}
$$

with

$S(z)=\left[(1+z)^{a \eta}+\left(\frac{1+z}{B}\right)^{b \eta}+\left(\frac{1+z}{C}\right)^{c \eta}\right]^{1 / \eta}$,

where $a=3.4, b=-0.3, c=-3.5, \eta \approx$ $-10, B \simeq 5000$, and $C \simeq 9$ (Hopkins \& Beacom 2006; Yüksel et al. 2008).

The sum in Eq. (8) is over the different galactic and cluster/group contributions. We assume a fraction $\xi_{s b g}$ of neutrinos are produced in starburst galaxies with the rest $\xi_{s f g}=1-\xi_{s b g}$ produced in normal starforming galaxies:

$$
\begin{array}{r}
f_{p p, s b g}=\xi_{s b g}\left(1-e^{-\tau_{p p, g, s b g}}\right) \\
f_{p p, s f g}=\xi_{s f g}\left(1-e^{-\tau_{p p, g, s f g}}\right) \\
f_{p p, c l}=\left(1-e^{-\tau_{p p, c l}}\right) \times
\end{array}
$$
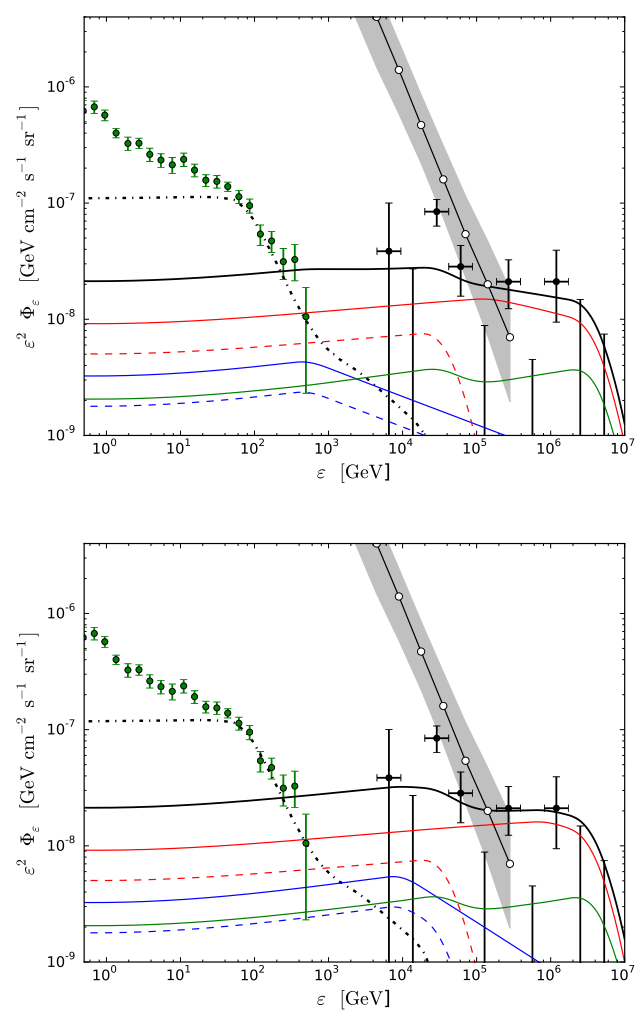

FIG. 1.- Diffuse flux per flavor of neutrinos (solid black) and $\gamma$-rays (dash-dot) from $\mathrm{HNe}$ and $\mathrm{SNe}$, for a diffusion coefficient (top): $D \propto \varepsilon_{p}^{1 / 3}$, (bottom): $D \propto \varepsilon_{p}^{1 / 2}$, in both the host galaxy and cluster. For both figures $\mathrm{HNe}$ and SNe release on average $2.8 \times 10^{51}$ and $4.8 \times 10^{49} \mathrm{ergs}$ of CR energy respectively, and the proton spectral index is $\Gamma=2$. The black line with white circles denotes the measured flux of atmospheric neutrinos (Abbasi et al. 2011). The starburst galaxy scale height, density, and magnetic field strength are $H_{s b g}=300 \mathrm{pc}, n_{s b g}=200 \mathrm{~cm}^{-3}$, and $B_{s b g}=200 \mu \mathrm{G}$ and are represented by red lines. For normal star-forming galaxies $H_{s f g}=1000 \mathrm{pc}, n_{s f g}=$ $1 \mathrm{~cm}^{-3}$, and $B_{s f g}=6 \mu \mathrm{G}$; they are represented by blue lines. The contribution from $\mathrm{HNe}$ are marked with solid lines colored while those from the $\mathrm{SNe}$ are dashed. The solid green line denotes the total cluster contribution (i.e. $\mathrm{HNe}$ and $\mathrm{SNe}$ from both types of galaxies). Green data points correspond to the Fermi measurements of the extragalactic diffuse $\gamma$-ray background (Ackermann et al. 2014). Black points correspond to the IceCube measurements of astrophysical neutrinos (Aartsen et al. 2015), note that two of the low energy data points are within the gray lines of the error bars of the atmospheric flux.

$$
\left[\xi_{s b g} e^{-\tau_{p p, g, s b g}}+\xi_{s f g} e^{-\tau_{p p, g, s f g}}\right]
$$

Note that in the last line of Eq. (11) only CRs which escape from the galaxies can contribute to the cluster component.

For our cluster/group parameters and the average galaxy parameters taken in 84 , the diffuse neutrino flux per flavor for a $D \propto \varepsilon_{p}^{1 / 3}$ diffusion coefficient is shown in Fig. 11 (top panel). Here 


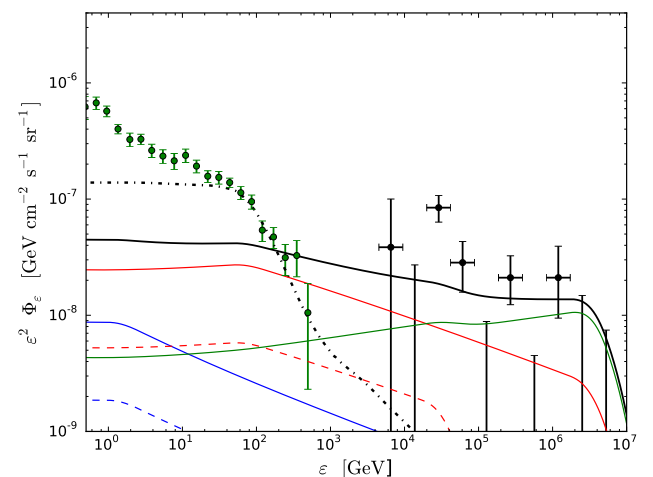

Fig. 2.- Diffuse flux per flavor of neutrinos (solid black) and $\gamma$-rays (dash-dot) from $\mathrm{HNe}$ and $\mathrm{SNe}$ which release on average $7.5 \times 10^{51}$ and $5 \times 10^{49}$ ergs of CR energy respectively, with a phenomenologically motivated diffusion coefficient based on observations of CR diffusion in the Milky Way galaxy (see text for details). In this case, cluster contributions are dominant at high energies.

the contributions of the $p p$ interactions in the galaxies are indicated both for the supernova and hypernova components. In the same figure, the resulting diffuse $\gamma$-ray flux is also shown, resulting from the corresponding $\pi^{0}$ decays and subsequent pair cascades in the intergalactic medium, which are discussed in 8 . A similar calculation for a $D \propto \varepsilon_{p}^{1 / 2}$ Komolgoroff type diffusion coefficient is shown in the bottom panel of Fig. 1.

The situation depends strongly on the diffusion coefficients of both galaxies and clusters, which are uncertain especially at high energies; and, due in large part to uncertainties in the magnetic coherence length. For example, the diffusion coefficient for normal galaxies used in Fig. 1 is 10 times lower than the value obtained for our Milky Way. While this discrepancy is alleviated by inhomogeneities, the diffuse Galactic emission suggests that the CR spectral break is much lower since the observed $\gamma$-ray spectrum is already steep at GeV energies (Ackermann et al. 2012a). In Fig. 2, we conservatively use the diffusion coefficient suggested for our Milky Way by Blasi \& Amato (2012) for normal star-forming galaxies. We then use the scaling relation $D \propto r_{L}\left(\varepsilon_{p}\right)^{\alpha}$ (see $\$ 4$ ) to determine the diffusion coefficient in starbursts. Since the break energy is sensitive to the diffusion coefficient, one sees that the diffuse neutrino background cannot be explained by star-forming galaxies in this case, even with an optimistically high fraction of HN kinetic energy converted to CRs (i.e. $7.5 \times 10^{51} \mathrm{erg}$ ). At high energies the galaxy contribution may not be appreciable. At and below $\varepsilon_{p *, g} \simeq 1.11 \times 10^{18} \ell_{g, 20} B_{g,-3.7} \mathrm{eV}$, however, the galactic contribution becomes considerable, $\tau_{p p, g}\left(\varepsilon_{p *, g}\right) \simeq 0.33$, overcoming the
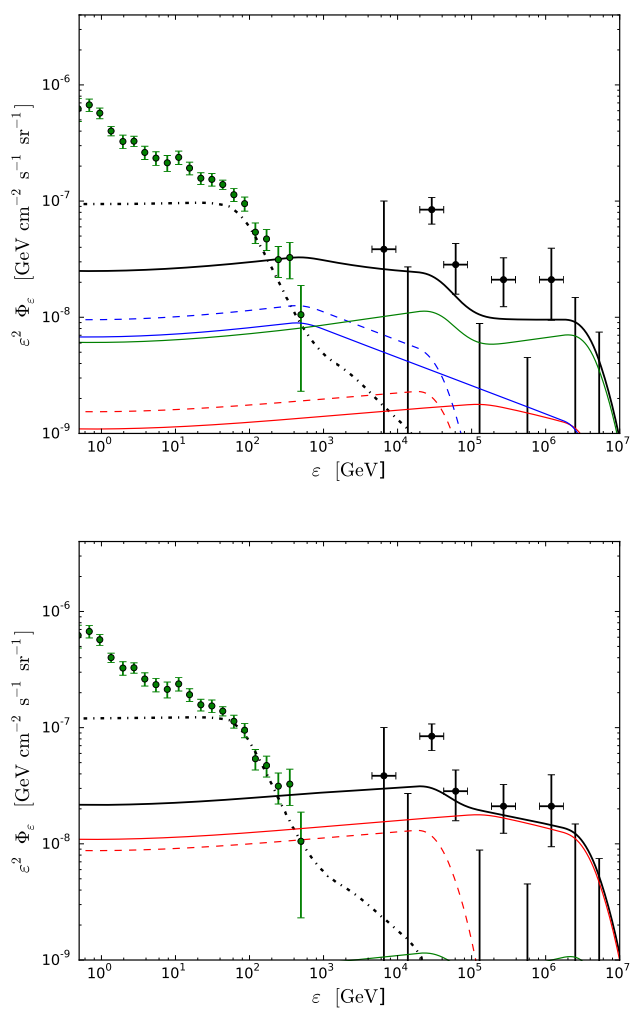

FIG. 3. - Same as Fig. 1 with $D \propto \varepsilon_{p}^{1 / 3}$ but top: $\xi_{s b g}=0.01$ with $E_{c r, h n}=5 \times 10^{51} \mathrm{erg}$, and $E_{c r, s n}=$ $2.2 \times 10^{50}$ erg bottom: $\xi_{s b g}=0.5$ with $E_{c r, h n}=10^{51} \mathrm{erg}$, and $E_{c r, s n}=2.5 \times 10^{49} \mathrm{erg}$

cluster contribution at the same energy. For this combination of parameters the cluster and group contribution should be dominant, and it is possible to explain the hard spectrum of the diffuse neutrino background. Note that the parameters used for the cluster/group contribution to the diffuse neutrino flux are optimistic, and massive clusters are only a fraction of the total cluster population.

Returning to the parameters used in Fig. 1 . the flux resulting from average host galaxies with a smaller (top) and larger (bottom) fraction of CRs produced in starburst galaxies is shown in Fig. 3. Here the fraction of HN/SN CR energy was adjusted ad-hoc in order to fit the observed neutrino flux with $E_{c r, h n}=5 \times 10^{51} \mathrm{erg}$ and $E_{c r, s n}=2.2 \times 10^{50} \mathrm{erg}$ for $\xi_{s b g}=0.01$, and $E_{c r, h n}=10^{51} \mathrm{erg}$ and $E_{c r, s n}=2.5 \times 10^{49} \mathrm{erg}$ for $\xi_{s b g}=0.5$ respectively. The diffusion coefficient was taken to be $D \propto \varepsilon_{p}^{1 / 3}$ while leaving the remaining parameters unchanged.

The Fig. 1 and 3 were calculated for "typical" star-forming galaxies with parameters as given 

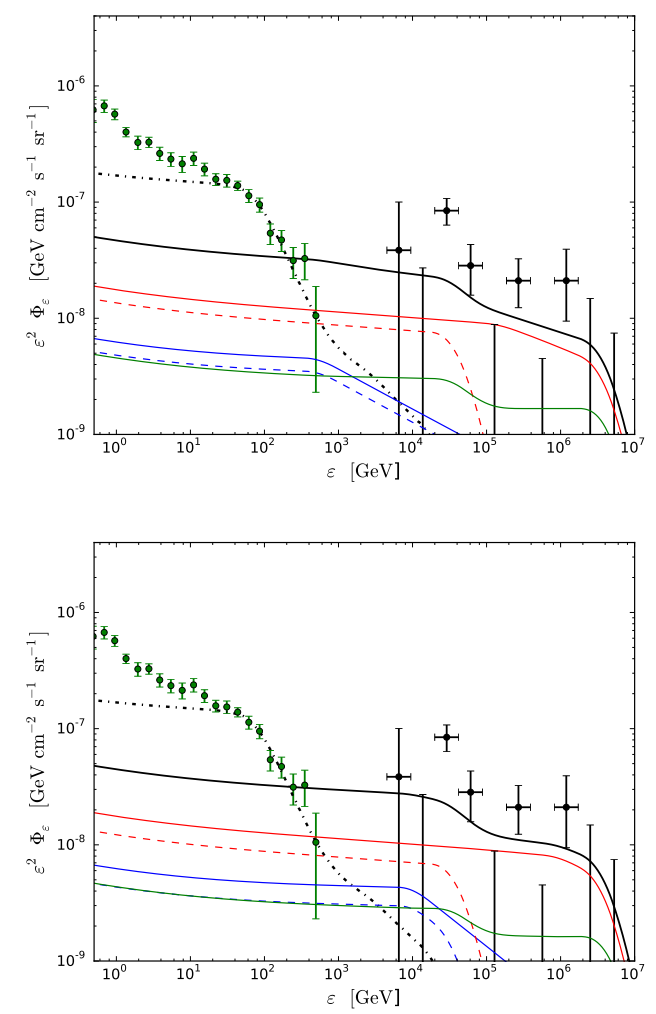

FIG. 4. - Same as Fig. 11 but for a proton index $\Gamma=2.1$, $E_{c r, h n}=3.5 \times 10^{51} \mathrm{erg}$, and $E_{c r, s n}=10^{50} \mathrm{erg}$. top: $D \propto \varepsilon_{p}^{1 / 3}$, bottom: $D \propto \varepsilon_{p}^{1 / 2}$.

above, and for a proton injection spectrum $\Gamma=2$. We consider next the SFG and SBG contributions using the same parameters, but with a proton injection index $\Gamma=2.1$, results are shown in Fig. 4

The effect of $p \gamma$ interactions in the galactic and intracluster medium is sub-dominant relative to the $p p$ collisions in the relevant energy range, although it becomes dominant at very high energies (Kotera et al. 2009).

\section{GAMMA-RAY CASCADES}

The same $p p$ interactions which produce neutrinos also produce high-energy $\gamma$-rays with typical energy $\epsilon_{\gamma} \sim \varepsilon_{p} / 10$. Note that because of this connection, their resulting flux can be related by $\epsilon_{\gamma}^{2} \Phi_{\epsilon_{\gamma}}=\left.2^{\Gamma-1} \varepsilon_{\nu}^{2} \Phi_{\varepsilon_{\nu}}\right|_{\varepsilon_{\nu}=0.5 \epsilon_{\gamma}}$. When $\gamma$-rays with energy $\epsilon_{\gamma}^{\prime} \gtrsim 100 \mathrm{GeV}$ are injected into intergalactic space sufficiently far from Earth (i.e. $\sim$ few Mpc), they undergo $\gamma \gamma$ interactions with extragalactic background light (EBL) photons producing electron/position pairs. The pairs scatter additional EBL photons via the inverse Compton mechanism generating an electromag- netic cascade. The resulting cascaded $\gamma$-ray spectrum takes a universal form, (e.g., Berezinskii \& Smirnov 1975, Murase et al. 2012):

$$
\epsilon_{\gamma} \frac{d N_{\gamma}}{d \epsilon_{\gamma}} \propto G_{\epsilon_{\gamma}}= \begin{cases}\left(\frac{\epsilon_{\gamma}}{\epsilon_{\gamma}^{b^{r}}}\right)^{-1 / 2} & \epsilon_{\gamma} \leq \epsilon_{\gamma}^{b r} \\ \left(\frac{\epsilon_{\gamma}}{\epsilon_{\gamma}^{b r}}\right)^{1-s} & \epsilon_{\gamma}^{b r}<\epsilon_{\gamma} \leq \epsilon_{\gamma}^{c u t}\end{cases}
$$

The characteristic energies $\epsilon_{\gamma}^{c u t}$ and $\epsilon_{\gamma}^{b r}$ given above are defined by $1=\tau_{\gamma \gamma}\left[\epsilon_{\gamma}^{c u t}, z\right]$ and $\epsilon_{\gamma}^{b r}=$ $0.0085(1+z)^{2}\left(\frac{\epsilon_{\gamma}^{\text {cut }}}{0.1 \mathrm{TeV}}\right)^{2}$ respectively and the high-energy spectral index is generally taken to be $s \sim 2$. Here $\tau_{\gamma \gamma}$ is the optical depth for a high energy photon traveling through intergalactic space, the values for which are from model $\mathrm{C}$ of Finke et al. (2010).

There is also an attenuated component to the observed $\gamma$-ray flux from photons with energy $\epsilon_{\gamma} \lesssim \epsilon_{\gamma}^{c u t}$ which can be calculated similarly to eq. 8

$$
\begin{aligned}
& \epsilon_{\gamma}^{2} \Phi_{\gamma}^{\text {unatt }}=\frac{c}{4 \pi} \int d z\left|\frac{d t}{d z}\right| e^{-\tau_{\gamma \gamma}\left[(1+z) \epsilon_{\gamma}, z\right]} \times \\
& \frac{1}{(1+z)^{4}}\left[\frac{2^{\Gamma-2}}{3} \sum_{i} f_{i, p p}\left(\varepsilon_{p} Q_{\varepsilon_{p}}\right)_{p h y s}\right]_{\varepsilon_{c r}^{\prime}=10(1+z) \epsilon_{\gamma}}
\end{aligned}
$$

which combined with Eq. 12 can be compared with Fermi-LAT measurements of the extragalactic diffuse $\gamma$-ray background (Ackermann et al. 2014). Figures 1 through 4 show our calculated diffuse flux of neutrinos and $\gamma$-rays along with data from IceCube and Fermi.

\section{DISCUSSION AND SUMMARY}

In this work, we discuss the starburst scenario in light of the new $10 \mathrm{TeV}$ neutrino data. Although there are large systematic uncertainties involved in removing the atmospheric muon background at such low energies Aartsen et al. 2014a), it may be challenging to explain the diffuse neutrino flux in the whole energy range with a single power-law component with $\Gamma \sim 2$. Adding the SNe contribution enables us to explain the low-energy data, but we find that constraints from the diffuse $\gamma$-ray background are quite stringent (Murase et al. 2013). If the CR energy input by SNe is a factor of two larger than that by HNe, the diffuse $\gamma$-ray background is violated. Additional constraints could be placed on the cluster contribution by considering the concomitant radio emission, although in the strong evolution case these limits are weaker than the ones imposed by the accretion shock scenario 
(Zandanel et al. 2014). From the $\gamma$-ray limits, we conclude that if the diffuse neutrino background in the $\mathrm{PeV}$ range originates mainly from $\mathrm{HNe}$ (and their host galaxies), the HN contribution should be larger than or at least comparable to the SN contribution. However, interestingly, in cases where the cluster/group contribution is mainly responsible for the diffuse neutrino flux, it is still possible for the SN contribution to overwhelm the HN contribution (e.g. the top panel of Fig. 3).

The strong case scenario, where the $\sim 10 \mathrm{TeV}$ neutrino data are explained by CR reservoirs, has an interesting feature that can be tested soon. As proposed by Murase et al. (2013), CR reservoirs can give a common explanation for both the diffuse neutrino and $\gamma$-ray backgrounds. In general, the contributions from starbursts and other sources to the neutrino flux above $100 \mathrm{TeV}$ result in subdominant contributions to the diffuse $\gamma$-ray background. However, as we show above any source which contributes significantly to the $10 \mathrm{TeV}$ diffuse neutrino flux in the $p p$ scenario must also account for almost all of the diffuse $\gamma$ ray background. It is commonly believed that the diffuse $\gamma$-ray background is dominated by unresolved blazars (Inoue 2014; Di Mauro et al. 2014), implying a comparatively smaller starburst contribution. Although there are still significant uncertainties in the modeling of both blazar (e.g., Ajello et al. 2014, 2012) and starburst contributions (e.g., Ackermann et al. 2012b, Tamborra et al. 2014), our results imply that the strong case scenario can be tested by an improved understanding or characterization of the diffuse $\gamma$-ray background.

If, for example, it is proven that blazars are responsible for $\gtrsim 50 \%$ of the observed diffuse $\gamma$-ray background, the starburst contribution to the diffuse neutrino background at low energies should be small, especially if the CR energy input from SNe is comparable to or larger than that from HNe. Specifically, tighter constraints on the the unresolved blazar fraction of the diffuse $\gamma$-ray background measured by Fermi and possibly the High Altitude Water Cherenkov (HAWC) observatory (BenZvi 2015), as well as the low energy $\left(\varepsilon_{\nu} \lesssim 100 \mathrm{TeV}\right)$ spectral shape of the astrophysical neutrino flux as analyzed by Aartsen et al. (2014a), will impose limits to the corresponding fluxes from galaxy clusters/groups. If there is little room for the $\mathrm{CR}$ reservoirs, other sources need to be responsible for the low-energy neutrino component. For instance, there might be a significant contribution from Galactic sources. Although the Galactic diffuse emission by CRs propagating in our Milky Way cannot provide the main contribution Ahlers \& Murase 2014;
Joshi et al. 2014), some extended sources such as the Fermi Bubbles Ahlers \& Murase 2014; Lunardini et al. 2014) or nearby HN remnants (Fox et al. 2013; Ahlers \& Murase 2014) could be viable. Alternatively, the diffuse neutrino background might be produced mainly by hidden neutrino sources via $p \gamma$ processes (Murase \& Ioka 2013 Kalashev \& Troitsky 2014: Kimura et al. 2014). The advantage of the strong case considered here is that it can be tested by multimessenger approaches. It has been commonly believed that Galactic CRs come from SN remnants. If the diffuse neutrino background is dominated by star-forming galaxies, our results imply that even Galactic CRs may include significant contributions from past HN remnants.

CR acceleration to energies $\gtrsim 10^{16}-10^{17} \mathrm{eV}$ has also been proposed in other accelerators, such as shocks in AGN jets, (e.g., Hillas 1984; Biermann \& Strittmatter 1987; Dermer et al. 2014), AGN winds (Murase et al. 2014; Tamborra et al. 2014), and AGN cores (Stecker et al. 1991; Alvarez-Muñiz \& Mészáros 2004 Kimura et al. 2014). While in such cases neutrinos can come from AGNs themselves, CRs escaping from AGNs can also produce neutrinos in intergalactic space, which may give a significant contribution to the diffuse neutrino background (Murase et al. 2013). Other possibilities are accretion shocks onto clusters of galaxies (Murase et al. 2008a; Dobardzic \& Prodanovic 2014), galaxy mergers in clusters (Kashiyama \& Mészáros 2014), and $\gamma$-ray bursts including trans-relativistic SNe or low-luminosity $\gamma$-ray bursts (Murase et al. 2008b, Wang et al. 2007). In principle, the discussion of the cluster/group propagation effects discussed above also applies to any intracluster sources. The main difference between these other sources and $\mathrm{SNe} / \mathrm{HNe}$ (or sources inside galaxies in general) is that the CRs accelerated in the former do not undergo $p p$ interactions in the galactic gas, but only in the intracluster gas; whereas CRs from HNe and galactic sources undergo $p p$ interactions in both the host galaxy and the cluster/group. This disparity may be relevant at sub-PeV and $\mathrm{TeV}$ energies, where the spectral shape of the neutrino flux can provide clues to the source. In this energy range we expect the advective escape and Hubble times to dominate the galactic and cluster diffusion times respectively, and at different critical energies (i.e. $\varepsilon_{w}$ and $\varepsilon_{p, c l}$ as in $\$ 4$. Therefore, for galactic sources (that are extragalactic), a soft spectrum is typically expected at energies below the maximum acceleration energy, with $\varepsilon_{\nu}^{2} \Phi_{\nu} \propto \varepsilon_{\nu}^{-\alpha}$ (for a diffusion time $\propto \varepsilon_{\nu}^{-\alpha}$ ), with a leveling off of the slope to $\varepsilon_{\nu}^{2} \Phi_{\nu} \sim$ constant below about $\varepsilon_{\nu, g} \sim 130(2 /(1+z)$ ) TeV (assuming, e.g., a galactic magnetic field strength of 
$200 \mu \mathrm{G}$ and diffusion exponent $\alpha=1 / 2$ ). Sources which release their CRs directly into the intracluster medium on the other hand, are expected to produce relatively flat neutrino spectra below a break around $\varepsilon_{\nu, c l} \sim$ few $(2 /(1+z)) \mathrm{PeV}$, steepening above that. Such a spectral softening of the cluster contribution is not seen for the $\mathrm{HNe}$ model considered here (e.g. in Fig. 2 because the break energy due to diffusion is lower than the cutoff energy imposed by maximally accelerated CRs. While both $\varepsilon_{\nu, g}$ and $\varepsilon_{\nu, c l}$ are subject to large uncertainties in parameters, the presence of their corresponding features could be suggestive of sources which are embedded in star-forming or starburst galaxies.

As shown by Murase et al. (2013), in the $p p$ scenario, the neutrino spectrum cannot be softer than about $\varepsilon_{\nu}^{2} \Phi_{\nu} \propto \varepsilon_{\nu}^{-0.2}$ at low energies for the corresponding $\gamma$-ray component not to violate the Fermi measurements of the diffuse $\gamma$-ray background (Ackermann et al. 2014). At the same time, a flat spectrum at moderate to high energies creates tension with the non-detection of neutrinos with energies near the Glashow resonance at $\varepsilon_{\nu} \sim 6 \mathrm{PeV}$, which necessitates a neutrino spectral shape near that energy steeper than $\varepsilon_{\nu}^{2} \Phi_{\nu} \propto \varepsilon_{\nu}^{-0.3}$ (Laha et al. 2013). Such a spectral break can occur if acceleration stops or there is a transition in the diffusive escape time (i.e. $\left.D\left(\varepsilon_{c r}\right) \propto \varepsilon_{c r}^{\alpha} \rightarrow D\left(\varepsilon_{c r}\right) \propto \varepsilon_{r r}^{2}\right)$ around $\varepsilon_{c r} \sim 240((1+z) / 2) \mathrm{PeV}$ (e.g., Murase et al. 2013 Anchordoqui et al. 2014). As can be seen in Fig. 1 and 3 the model presented here can also resolve this tension. The neutrino spectrum is flat at low energies $\varepsilon_{\nu} \lesssim 130 \mathrm{TeV}$ and softens to $\varepsilon_{\nu}^{2} \Phi_{\nu} \propto \varepsilon_{\nu}^{-\alpha}$ slightly before the Glashow resonance, while the corresponding diffuse $\gamma$-ray spectrum is below the Fermi measured flux.

We have shown in $\$ 5$ that the high-energy diffuse neutrino flux could potentially be explained by $\mathrm{HNe}$, predominantly those located in dense starburst galaxies (e.g. the red solid curve in the bottom panel of Fig. 1) especially for $\varepsilon_{\nu} \gtrsim 50$ $\mathrm{TeV}$. For neutrinos with this energy and below, the SNe in both starburst and normal starforming galaxies contribute significantly to the diffuse flux and produce a "bump" in the spectrum Reasonable fits by eye are obtained for the diffuse neutrino flux measured by IceCube including the latest $\mathrm{TeV}$ data by using reasonable parameters for the sources as well as the diffusion properties in hosting structures. Such a flux also approximates but does not violate the diffuse $\gamma$-ray background measured by Fermi. This does not mean that $\mathrm{SNe}$ and HNe are necessarily the only sources contributing to the neutrino and $\gamma$-ray diffuse backgrounds. It supports, however, the case for CR reservoirs such as clusters and groups being promising sources which could contributes at least a significant fraction of these backgrounds, without violating both CR (Yoshida \& Takami 2014) and $\gamma$-ray constraints.

This work was partially supported by NASA NNX13AH50G. We are grateful to J. van Santen for providing the IceCube data points.

\section{REFERENCES}

Aartsen, M., Ackermann, M., Adams, J., et al. 2015, Physical Review D, 91, 022001

Aartsen, M. G., Abbasi, R., Abdou, Y., et al. 2013, Physical Review Letters, 111, 021103

Aartsen, M. G., Ackermann, M., Adams, J., et al. 2014a, ArXiv e-prints, 1410.1749, arXiv:1410.1749

—. 2014b, Physical Review Letters, 113, 101101

Abbasi, R., Abdou, Y., Abu-Zayyad, T., et al. 2011, Phys.Rev.D , 83, 012001

Ackermann, M., Ajello, M., Atwood, W. B., et al. 2012a, Astrophys.J., 750, 3

Ackermann, M., Ajello, M., Allafort, A., et al. 2012b, Astrophys.J., 755, 164

Ackermann, M., Ajello, M., Albert, A., et al. 2014, arXiv preprint arXiv: 1410.3696

Ahlers, M., \& Murase, K. 2014, Phys.Rev.D , 90, 023010

Ajello, M., Shaw, M. S., Romani, R. W., et al. 2012, Astrophys.J., 751, 108

Ajello, M., Romani, R. W., Gasparrini, D., et al. 2014, Astrophys.J., 780, 73

Alvarez-Muñiz, J., \& Mészáros, P. 2004, Phys.Rev.D, 70, 123001

$\ddagger$ As we were preparing to submit our calculation, a similar qualitative conclusion was posted by Chakraborty \& Izaguirre (2015), who however did not consider the diffuse $\gamma$-ray constraints from Fermi observations that are very important.
Anchordoqui, L. A., Paul, T. C., da Silva, L. H. M., Torres, D. F., \& Vlcek, B. J. 2014, Phys.Rev.D , 89, 127304

Arcavi, I., Gal-Yam, A., Kasliwal, M. M., et al. 2010, Astrophys.J., 721, 777

Beck, R. 2008, in American Institute of Physics Conference Series, Vol. 1085, American Institute of Physics Conference Series, ed. F. A. Aharonian, W. Hofmann, \& F. Rieger, 83-96

BenZvi, S. 2015, Physics Procedia, 61, 399

Berezinskii, V. S., \& Smirnov, A. I. 1975, Ap\&SS, 32, 461

Berezinsky, V. S., Blasi, P., \& Ptuskin, V. S. 1997, Astrophys.J., 487, 529

Biermann, P. L., \& Strittmatter, P. A. 1987, Astrophys.J., 322, 643

Blasi, P., \& Amato, E. 2012, Jour. Cosmology and Astro-Particle Phys. , 1, 11

Chakraborty, S., \& Izaguirre, I. 2015, ArXiv e-prints, arXiv:1501.02615

Chang, X.-C., \& Wang, X.-Y. 2014, ArXiv e-prints, 1406.1099, arXiv:1406.1099

Crocker, R. M. 2012, M.N.R.A.S., 423, 3512

Dermer, C. D., Murase, K., \& Inoue, Y. 2014, Journal of High Energy Astrophysics, 3, 29

Di Mauro, M., Cuoco, A., Donato, F., \& Siegal-Gaskins, J. M. 2014, Jour. Cosmology and Astro-Particle Phys., 11, 21

Dobardzic, A., \& Prodanovic, T. 2014, ArXiv e-prints, arXiv: 1412.5678 
Drury, L. 2011, Monthly Notices of the Royal Astronomical Society, 415, 1807

Drury, L. O. 1983, Reports on Progress in Physics, 46, 973

Finke, J. D., Razzaque, S., \& Dermer, C. D. 2010 Astrophys.J., 712, 238

Fox, D. B., Kashiyama, K., \& Mészáros, P. 2013, Astrophys.J., 774, 74

Guetta, D., \& Della Valle, M. 2007, Astrophys.J.Lett., 657, L73

He, H.-N., Wang, T., Fan, Y.-Z., Liu, S.-M., \& Wei, D.-M. 2013, Phys.Rev.D, 87, 063011

Hillas, A. M. 1984, Annu.Rev.Ástron.Astrophys. , 22, 425

Hopkins, A. M., \& Beacom, J. F. 2006, Astrophys.J., 651,142

IceCube Collaboration. 2013, Science, 342, arXiv: 1311.5238

Inoue, S., Aharonian, F. A., \& Sugiyama, N. 2005, Astrophys.J.Lett. , 628, L9

Inoue, Y. 2014, ArXiv e-prints, arXiv:1412.3886

Joshi, J. C., Winter, W., \& Gupta, N. 2014, M.N.R.A.S. 439,3414

Kalashev, O. E., \& Troitsky, S. V. 2014, ArXiv e-prints, arXiv: 1410.2600

Kashiyama, K., \& Mészáros, P. 2014, Astrophys.J.Lett., 790, L14

Katz, B., Waxman, E., Thompson, T., \& Loeb, A. 2013, ArXiv e-prints, arXiv:1311.0287

Keeney, B. A., Danforth, C. W., Stocke, J. T., et al. 2006, Astrophys.J., 646, 951

Kelner, S. R., Aharonian, F. A., \& Bugayov, V. V. 2006 , Phys.Rev.D, 74, 034018

Keshet, U., Waxman, E., \& Loeb, A. 2004, Astrophys.J. 617,281

Kimura, S. S., Murase, K., \& Toma, K. 2014, ArXiv e-prints, arXiv:1411.3588

Kotera, K., Allard, D., Murase, K., et al. 2009, Astrophys.J., 707, 370

Laha, R., Beacom, J. F., Dasgupta, B., Horiuchi, S., \& Murase, K. 2013, Phys.Rev.D , 88, 043009

Liu, R.-Y., \& Wang, X.-Y. 2013, Astrophys.J., 766, 73

Liu, R.-Y., Wang, X.-Y., Inoue, S., Crocker, R., \& Aharonian, F. 2014, Phys.Rev.D , 89, 083004

Loeb, A., \& Waxman, E. 2006, Jour. Cosmology and Astro-Particle Phys., 5, 3
Lunardini, C., Razzaque, S., Theodoseau, K. T., \& Yang, L. 2014, Phys.Rev.D , 90, 023016

Mészáros, P. 2014, Nuclear Physics B Proceedings Supplements, 256, 241

Murase, K. 2014, ArXiv e-prints, 1410.3680, arXiv: 1410.3680

Murase, K., Ahlers, M., \& Lacki, B. C. 2013, Phys.Rev.D , 88, 121301

Murase, K., Beacom, J. F., \& Takami, H. 2012, Journal of Cosmology and Astroparticle Physics, 2012, 030

Murase, K., Inoue, S., \& Nagataki, S. 2008a Astrophys.J.Lett., 689, L105

Murase, K., Inoue, Y., \& Dermer, C. D. 2014, ArXiv e-prints, arXiv: 1403.4089

Murase, K., \& Ioka, K. 2013, Physical Review Letters, 111,121102

Murase, K., Ioka, K., Nagataki, S., \& Nakamura, T. 2008b, Phys.Rev.D , 78, 023005

Parizot, E. 2004, Nuclear Physics B-Proceedings Supplements, 136, 169

Smith, N., Li, W., Filippenko, A. V., \& Chornock, R. 2011, M.N.R.A.S., 412, 1522

Stecker, F. W., Done, C., Salamon, M. H., \& Sommers, P. 1991, Physical Review Letters, 66, 2697

Strickland, D. K., \& Heckman, T. M. 2009, Astrophys.J., 697, 2030

Sveshnikova, L. G. 2003, Astron.Astrophys. , 409, 799

Tamborra, I., Ando, S., \& Murase, K. 2014, Jour. Cosmology and Astro-Particle Phys., 9, 43

Taylor, M., Cinabro, D., Dilday, B., et al. 2014, Astrophys.J., 792,135

Wang, X.-Y., Razzaque, S., Mészáros, P., \& Dai, Z.-G. 2007, Phys.Rev.D , 76, 083009

Waxman, E., \& Bahcall, J. 1997, Phys. Rev. Lett., 78, 2292

Yoshida, S., \& Takami, H. 2014, Phys.Rev.D, 90, 123012

Yüksel, H., Kistler, M. D., Beacom, J. F., \& Hopkins, A. M. 2008, Astrophys.J.Lett., 683, L5

Zandanel, F., Tamborra, I., Gabici, S., \& Ando, S. 2014 ArXiv e-prints, arXiv:1410.8697 